\title{
Introduction to \\ State-variable Analysis
}

\author{
P. F. BLACKMAN
}

Senior Lecturer,

Department of Electrical Engineering,

Imperial College of Science and Technology,

London 
(C) P. F. Blackman 1977

Softcover reprint of the hardcover 1st edition 1977

All rights reserved. No part of this publication may be reproduced or transmitted, in any form or by any means, without permission

First published 1977 by

THE MACMILLAN PRESS LTD

London and Basingstoke Associated companies in New York Dublin Melbourne Johannesburg and Madras

ISBN 978-1-349-01842-0 ISBN 978-1-349-01840-6 (eBook)

DOI 10.1007/978-1-349-01840-6

Typeset in IBM Press Roman by

PREFACE LTD

Salisbury, Wilts

This book is sold subject to the standard conditions of the Net Book Agreement. 


\section{Contents}

Preface $\quad$ v

1 Principles of State-variable Analysis 1

1.1 Matrix Representation of Differential Equations 2

1.2 State-vector Differential Equation 4

1.3 Transformation of State-vector Differential Equation 9

$\begin{array}{lll}1.4 & \text { Transition Matrix } & 14\end{array}$

Problems 22

2 Eigenvalues, Eigenvectors and Trajectories 25

2.1 Root-locus Investigation of Eigenvalues 25

2.2 Diagonal System 29

2.3 Eigenvectors 32

2.4 Eigenvectors and Trajectories 45

2.5 Complex Eigenvalues 49

2.6 Repeated Eigenvalues $\quad 57$

Problems 64

3 State-variable Representation of Transfer Functions 71

3.1 Transfer-function Representation 71

3.2 Transfer Functions for Closed-loop Systems 77

3.3 Multivariable Transfer Functions $\quad 84$

$\begin{array}{ll}\text { Problems } & 89\end{array}$

4 State-variable Feedback $\quad 95$

4.1 State-variable Feedback 95

4.2 Missing State Variables 101

4.3 Closed-loop Performance Characteristics 103 
4.4 Introduction of Additional Parameters

4.5 Matrix Relationships for Transfer-function Manipulation

5 Controllability, Observability and Observers

5.1 Observability, Controllability and Pole-Zero Cancellation

5.2 State Controllability

5.3 Observability

5.4 Controllability and Observability Examples

5.5 Output Controllability

5.6 Observers

5.7 Observer in a Closed-loop System

6.1 Discrete-time Systems

6.2 Time-domain Analysis of Purely Discrete Systems

6.3 Systems with Continuous Portions

$6.4 z$-transformation of State Equations

6.5 Application of State-variable Analysis

6.6 Controllability and Observability

7.1 Minimum-time-response Systems

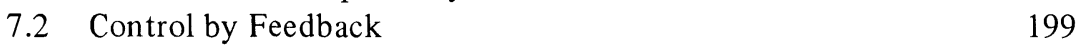

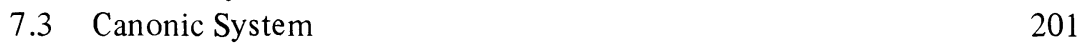

7.4 Application of $z$-transformation and Root-locus Method 205

7.5 Control of Triple Integrator and Undamped Oscillatory System 206

$\begin{array}{lll}7.6 & \text { Ripple-free Systems } & 213\end{array}$

$\begin{array}{ll}\text { Problems } & 218\end{array}$

Appendix 1 Initial-condition Zeros and Residue Evaluation in the s-plane

Appendix 2 Rank of a Matrix 


\section{Preface}

Many textbooks arise from lecture courses and this one had its origins in lectures given to control students in the Departments of Electrical Engineering and of Computing and Control. The lectures initially covered complex-frequency analysis, $s$-plane techniques and the root-locus method and gradually evolved over some years into using these techniques as starting points to introduce statevariable analysis. Another background feature was the development of laboratory experiments to illustrate many of the basic state-variable principles using simple analog computer equipment, the displayed results naturally leading to geometric interpretation. These two major background elements led to an approach to statevariable analysis with a very strong geometric and diagrammatic flavour together with the interpretation of state-variable phenomena in $s$-plane concepts. The main intention of this book is to use the above approach to establish a feel for the concepts involved and then develop the background of initial ideas rather than to cover the more advanced topics, which are very adequately treated in many texts.

Although the book is written in the context of control systems, the treatment of much of the introductory material - general principles (chapter 1), eigenvalues, eigenvectors and trajectories (chapter 2), transfer functions (initial portion of chapter 3) -is not specifically related to control ideas. The end of chapter 3 covers the state-variable representation of single-input single-output control systems, since at first experience the usual transfer-function forms and statevariable forms of such systems may be difficult to relate to each other. Statevariable feedback and its relationship to conventional compensation techniques and performance characteristics is covered in chapter 4 . The initial portion of chapter 5 treats controllability and observability with particular emphasis on obtaining an appreciation of the concepts involved and their interpretation from geometric and $s$-plane points of view. The final portion of the chapter gives an introduction to observer principles. The last two chapters, 6 and 7, cover discrete-time systems and the design of minimum-time-response and ripple-free systems. The designs use both state-variable and $z$-plane techniques to compare the methods. 
A knowledge of introductory control theory and Laplace transformation is assumed, together with an acquaintance with matrix algebra. Some particular aspects of Laplace transformation such as the representation of initial conditions by impulsive signals and by zeros in the $s$-plane and also graphical evaluation of residues are treated in appendix 1 . The rank of a matrix is covered in appendix 2 . An outline of $z$-transformation is given in appendix 3 to supplement the treatment given in chapter 6 .

To sum up, the book covers introductory ideas using a more intuitive as opposed to a mathematical approach. The general philosophy can be summarised in the following three statements (whatever aspect it is).

What does it look like as a block diagram?

Can it be interpreted geometrically?

If I can visualise it I can understand it.

In fact the above statements represent the canonic form for the book.

I am greatly indebted to colleagues in the Departments of Electrical Engineering and of Computing and Control for much helpful discussion, particularly to $\mathrm{Mr}$ J. M. Howl (Electrical Engineering) and $\mathrm{Mr} \mathrm{H}$. H. Johnson (Computing and Control). Finally I would like to record the unfailing patience and care of Mrs Magda Bicknell who typed the manuscript.

P. F. BLACKMAN 